\title{
Implementación del sensor de potencia del equipo Well Analyzer para el balanceo de unidades de bombeo mecánico balanceadas por aire
}

\section{Power sensor implementation of the Well Analyzer for the balancing of air balance rod pumping units}

\author{
Carlos Alberto Pérez ${ }^{1}$, Diego Fernando Córdoba² y Oscar Felipe Gaitán ${ }^{3}$
}

\begin{abstract}
Resumen
Se desarrolló un software que permite llegar adecuadamente a un balanceo de las unidades balanceadas por aire en el bombeo mecánico utilizadas en ECOPETROL, mediante la implementación del sensor de potencia del equipo Well Analyzer. Dicho sensor representa una herramienta muy útil, la cual permite una medida precisa y mucho más fiable que la usada actualmente y que permite realizar análisis de corriente del motor para llegar a determinar el rango en el que se debe tener la presión en el cilindro para balancear la unidad. Se llevaron a cabo pruebas de campo donde se tomaron mediciones de corriente variando la presión del cilindro para tratar de llegar a una aproximación de que proporción y relación existe específicamente entre la presión del cilindro y la corriente de la unidad motriz en el bombeo mecánico, que facilita el proceso de balanceo. En el desarrollo del software se utilizó Visual Studio y se escribió en el lenguaje C\# (C Sharp). Se utilizaron importantes librerías que permiten generar y encontrar funciones matemáticas necesarias para los análisis; se consiguió crear un software fácil de manejar, funcional y donde se lleva la secuencia para realizar un adecuado balanceo paso a paso integrando varios métodos que permiten tener un mejor criterio a la hora del balanceo en las unidades de bombeo mecánico balanceadas por aire. Por último se realizaron pruebas y validaciones para observar la eficiencia del programa.
\end{abstract}

\section{Palabras Clave}

Balanceo; Unidades Balanceadas por Aire; Visual Studio

\begin{abstract}
It was developed a software that allows to achieve a balancing properly balanced air units in the rod pumping used in ECOPETROL, by implementing the power sensor Well Analyzer team. This sensor is a useful tool which allows an accurate measurement and more reliable than the currently tool used and it allows us to make current analysis of the motor in order to determine the range in which it must have the cylinder pressure to balance the unit. It took several field tests where current measurements were made by varying the cylinder pressure to achieve an approximation among proportion and specifically the relationship between cylinder pressure and the flow of the power unit in the rod pumping, which facilitates us the balancing process. Software was developed in Visual Studio and it was programmed in C\# language. It was built owing to main libraries that allow for generating and finding mathematical functions required in the outcomes; we were able to create a user-friendly functional software, which has sequence for a proper balancing step integrating several methods that allow to have a better criterion at balancing the units. Finally, it carries out proofs and validations to observe the efficiency of the software.
\end{abstract}

\section{Keywords}

Balance; Balancing Air Units; Visual Studio

1 Ingeniero Electrónico. Docente Universidad Surcolombiana - Neiva. Av. Pastrana-Carrera 1. carpecc@yahoo.com

2 Ingeniero Electrónico. Universidad Surcolombiana - Neiva. Av. Pastrana-Carrera 1. diegocordoba@ingenieros.com

3 Ingeniero Electrónico. Universidad Surcolombiana - Neiva. Av. Pastrana-Carrera 1. oscarfgv@hotmail.com 


\section{Introducción}

El Bombeo mecánico es el método de levantamiento artificial más común, antiguo y ampliamente utilizado en la industria del petróleo. Usualmente es el más económico y fácil de mantener cuando es diseñado y operado apropiadamente (Svinos, 2005).

Incrementar la eficiencia del sistema de bombeo mecánico ha sido siempre importante. Razón por la cual ECOPETROL S.A. se ha interesado por realizar investigaciones que conlleven a la optimización de los procesos; pues grandes ahorros e incrementos en la producción son posibles mediante el uso de tecnología moderna e implementación de hardware para el diagnóstico de fallas en el pozo, tales como desgate de componentes del sistema, sobrecarga de la unidad motriz y en la caja reductora por desbalanceo del sistema.

Balancear una unidad es una tarea muy común y de gran importancia en la que se busca preservar el equilibrio energético durante el ciclo de bombeo; ya que una unidad fuera de balance puede sobrecargar el motor y la caja reductora generando fallas costosas y perdidas en la producción. Aunque en la práctica el balanceo perfecto es raramente alcanzado, es importante mantener la unidad tan cerca de estarlo como sea posible y para esto se debe hacer un análisis de torque o registrar un gráfico de corriente del motor en la carrera ascendente y descendente (Svinos, 2005).

Igualmente en el mismo ámbito de la optimización del balanceo de las unidades de bombeo mecánico, se han realizado estudios dirigidos al uso de la corriente y la potencia eléctrica para balancear unidades de bombeo mecánico (McCoy, 1993), análisis de corriente, potencia y torque para mejorar la eficiencia en bombeo mecánico (McCoy, 1992), reducción del consumo eléctrico en bombeo mecánico balanceando adecuadamente la unidad (Woods y McCoy 1995) y análisis en tiempo real de la corriente eléctrica para optimizar el sistema de bombeo mecánico (Podio et al. 1994).

Por tanto se estudiaron y evaluaron alternativas para realizar un balanceo óptimo en las unidades de tipo balanceadas por aire en bombeo mecánico, teniendo en cuenta métodos existentes tales como el eléctrico y el dinamométrico. De igual forma implementaron herramientas útiles con las que se cuentan actualmente y que no se aprovechan de la mejor forma; como lo es el sensor de potencia del equipo Well Analyzer.

\section{Metodología}

\subsection{Prueba y adquisición del dato de corriente y potencia}

Se instaló el sensor de potencia del equipo Well Analyzer, el cual corresponde a dos sensores de corriente y tres sensores de voltaje que permite adquirir la gráfica de corriente y potencia del motor en todo un ciclo de bombeo (ECHOMETER, 2006). Esta prueba se realizó en el pozo DT-38 perteneciente a campo DINA, el cual tiene una unidad de bombeo balanceada por aire A-320D-256-120, que tiene una capacidad de la caja reductora de 320000 Pulgas-Libras, una capacidad de la barra lisa de 25600 libras y una longitud de carrera de 120 pulgadas (LUFKIN, 2007).

Inicialmente se verificaron las condiciones de estado estable del pozo, realizando una prueba acústica de nivel de fluido y un dinagrama con la ayuda del equipo Well Analyzer y el software TWM. Seguidamente se instalaron los dos sensores de corriente y los tres sensores de voltaje verificando su adecuada instalación, como se observa en la figura 1. Una vez verificada la correcta instalación de los sensores, se adquirieron los datos de corriente y potencia.

Finalmente se obtuvo la gráfica de corriente y potencia del motor de la unidad de bombeo balanceada por aire del pozo DT-38. Como se observa en la figura 2. 


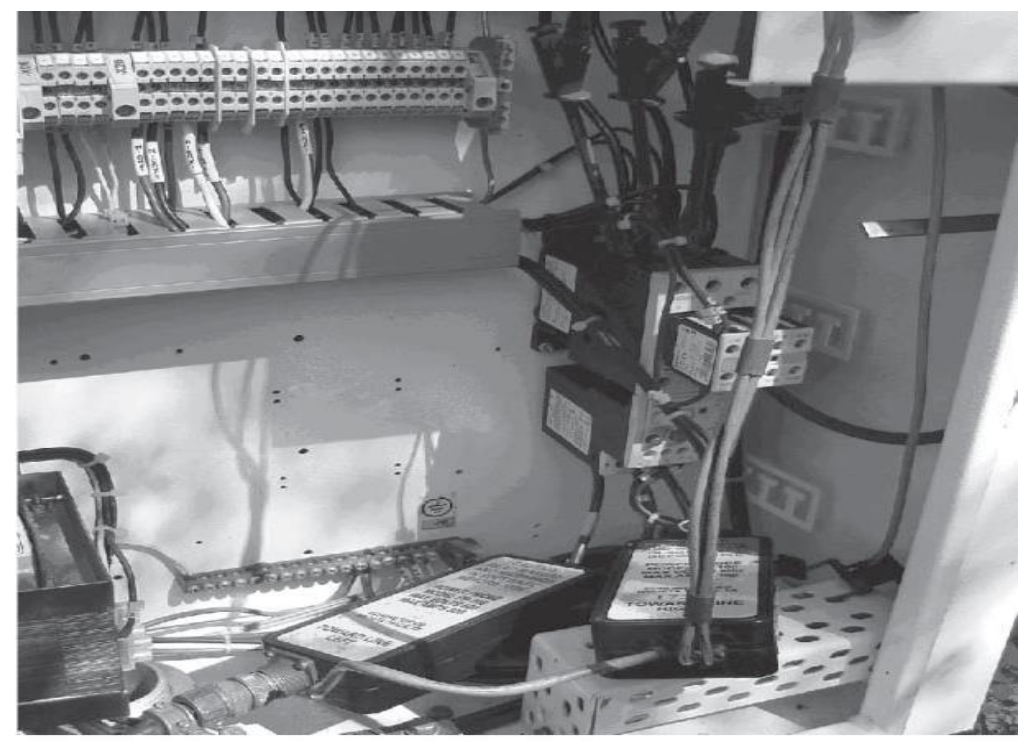

Figura 1. Instalación del sensor de potencia del Well Analyzer en el pozo DT-38

\subsection{Prueba de presión en el cilindro de unidades balanceadas por aire}

Se realizaron pruebas de presión en varios pozos del campo PALOGRANDE de ECOPETROL, con el fin de observar el comportamiento de la presión del cilindro que proporciona el contrabalance en las unidades balanceadas por aire, en diferentes posiciones de la manivela, es decir en diferentes posiciones de la cabeza de caballo de la unidad de bombeo. Cabe destacar que la mayoría de los pozos cuentan con sensor digital puesto en superficie para su comodidad en la lectura, pero existen pozos que cuentan únicamente con manómetro análogo que no arroja una información confiable y que además está pegado al cilindro, haciendo que la medida sea aún más difícil de tomar pues el manómetro sube y baja a medida que el cilindro lo hace.

Se definieron cinco puntos de medición, una en el punto de mayor presión (cabeza de caballo abajo), una en el punto de menor presión (cabeza de caballo arriba), una en la mitad del ciclo de bombeo y otras dos intermedias. Los datos medidos en diferentes pozos, son mostrados en la tabla 1.

Con los datos obtenidos, se generó una función matemática que describiera este comportamiento, es decir encontrar una curva tal que pasará por los puntos obtenidos ajustándose de la mejor manera. Posteriormente se llegó a una aproximación general del comportamiento de la presión del cilindro aprovechando los datos medidos en diferentes pozos. Esto se realizó con el fin de poder obtener la función de presión de cualquiera de estos pozos con tan sólo tener el valor máximo y mínimo de la presión existente en el cilindro; todo esto para realizar análisis de la presión que debe haber en el cilindro para que la unidad se encuentre balanceada. Para generalizar la función se llevaron a cabo varias pruebas, graficando inicialmente los datos experimentales obtenidos en pozo, y luego teniendo los valores máximos y mínimos de cada pozo se probó generando los demás puntos de diferentes formas y se encontró una aproximación donde es necesario tan sólo tener los puntos de presión máxima y mínima para generar la función de presión de cualquier pozo, aprovechando los datos de pozo medidos. Los resultados de estas aproximaciones se muestran en la figura 4, comprobando que fueron muy buenos.

\subsection{Balanceo de la unidad de bombeo}

Una vez verificadas las condiciones de estado estable de la unidad, se adquiere el dato de corriente y potencia del motor, mediante el sensor de potencia del equipo Well Analyzer, para ser posteriormente cargada y analizada en el software desarrollado, llamado ABU Balance.

Con los datos de corriente obtenidos se capturan tres puntos para cada medio ciclo, el primero de ellos en el momento que inicia cada medio ciclo, el segundo de ellos es el pico ó valor máximo dado y por último el valor en el fin de cada semiciclo y por medio de ajuste de curvas se obtiene una gráfica de corriente ideal como 
Se observa en la figura 3. Dicho ajuste de curvas inicialmente se realizó con la herramienta cftool (Curve Fitting Toolbox) de Matlab que permite encontrar y ajustar una curva en este caso polinómica de orden 2 que cumplía tales condiciones. Posteriormente se implementó una librería que contiene la aplicación de ajuste de curvas llamada Extrememathematics para Visual que logró suprimir el trabajo que hacía el MATLAB para dicho ajuste y mejorar la eficiencia del software desarrollado y facilidad de manejo para los operarios.

Posteriormente en el software desarrollado se solicita al usuario suministrar la presión máxima y la presión mínima del cilindro. Con estos dos datos y teniendo en cuenta los análisis de presión ya realizados, el mismo software hace un ajuste de curvas para encontrar la función en el tiempo que describe la presión del cilindro a lo largo de toda la trayectoria de desplazamiento de la cabeza de caballo. Encontrando una gráfica de presión como la mostrada en la figura 5 .

Luego el programa desarrollado procede a relacionar estas dos gráficas mediante el tiempo. Decide si es necesario extraer o suministrar aire al cilindro y de esta manera arroja la presión que permite una igualdad de picos de corriente en la gráfica de corriente o lo que es lo mismo, el balanceo de la unidad de bombeo.

Por cuestiones prácticas dar un valor exacto y constante en este tipo de sistemas es algo difícil, por tanto el Programa desarrollado arroja un rango de presión, para efecto de dar un poco de tolerancia al resultado obtenido.

Finalmente y con el objetivo de comprobar el adecuado balanceo de la unidad, se le pide al usuario volver a tomar datos de corriente obtenidos con la nueva presión que le suministró al cilindro, de acuerdo al rango de presión que se le sugirió mediante el software desarrollado. Obteniéndose una comparación de gráficas de corriente del motor antes y después del balanceo, al igual que los datos de interés del motor, como se ilustra en la figura 10.

\section{Resultados}

Se obtuvo entonces la gráfica de corriente y potencia del motor del pozo DT-38, mediante la implementación del sensor de potencia perteneciente al equipo Well Analyzer. Como se observa en la figura 2.

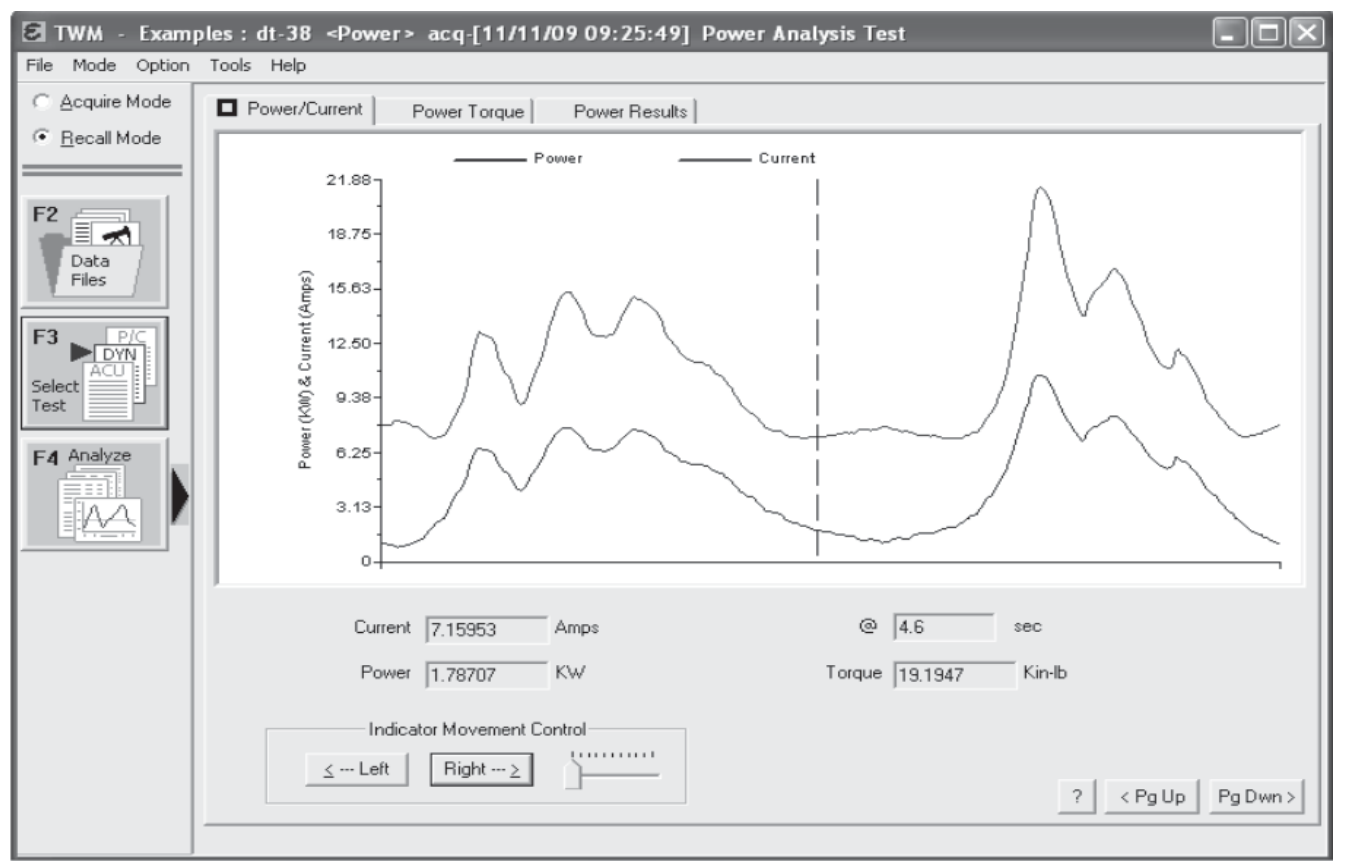

Figura 2. Gráfica de corriente y potencia del motor del pozo DT-38

A partir de la figura 2 y por medio de ajuste curvas se obtiene una corriente ideal o ajustada, donde solo se notan Los puntos de interés, como lo son los picos de corriente tanto en la subida como en la bajada del ciclo de bombeo 
Y de esta manera eliminar los cambios abruptos de corriente que afectan el análisis para realizar el balanceo. El resultado de esta operación es mostrado claramente en la figura 3.
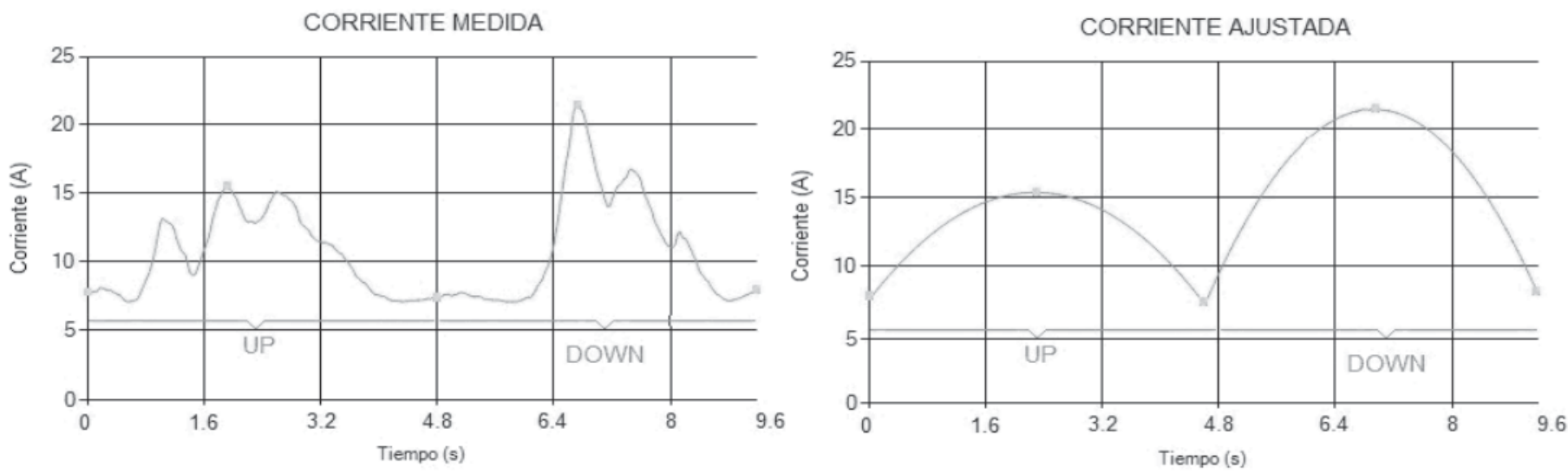

Figura 3. Puntos escogidos para la aproximación polinómica y gráfica de corriente ajustada por función Polinómica de orden 2, para el pozo DT-38

Posteriormente se obtuvieron los resultados de presión, los cuales se muestran en la tabla 1 y en la figura 3 , teniendo en cuenta que la palabra PG significa PALOGRANDE uno de los campos de ECOPETROL.

Tabla 1. Datos obtenidos de presión de los cilindros de los pozos PG-25, PG-12, PG-30, PG-08, PG-24

\begin{tabular}{|c|c|c|c|c|c|c|c|c|c|}
\hline \multirow{2}{*}{ Pozo } & \multirow{2}{*}{ Unidad } & \multirow{2}{*}{$\begin{array}{c}\text { Presión } \\
\text { máxima }\end{array}$} & Presión & \multirow{2}{*}{ mínima } & \multirow{2}{*}{ Rango } & \multicolumn{5}{|c|}{ Medidas } \\
\cline { 8 - 11 } & & & & $\mathbf{1}$ & $\mathbf{2}$ & $\mathbf{3}$ & $\mathbf{4}$ & $\mathbf{5}$ \\
\hline PG-25 & A-640D- 427-144 & 267 & 212 & 55 & 267 & 250 & 230 & 222 & 212 \\
\hline PG-12 & A-1280D-427-192 & 303 & 233 & 70 & 300 & 271 & 245 & 238 & 233 \\
\hline PG-30 & A-1280D-427-192 & 272 & 224 & 48 & 272 & 255 & 238 & 233 & 224 \\
\hline PG-08 & A-1280D-427-192 & 248 & 217 & 31 & 248 & 236 & 230 & 222 & 217 \\
\hline PG-24 & A-2560D-470-240 & 381 & 310 & 71 & 381 & 354 & 335 & 317 & 310 \\
\hline
\end{tabular}
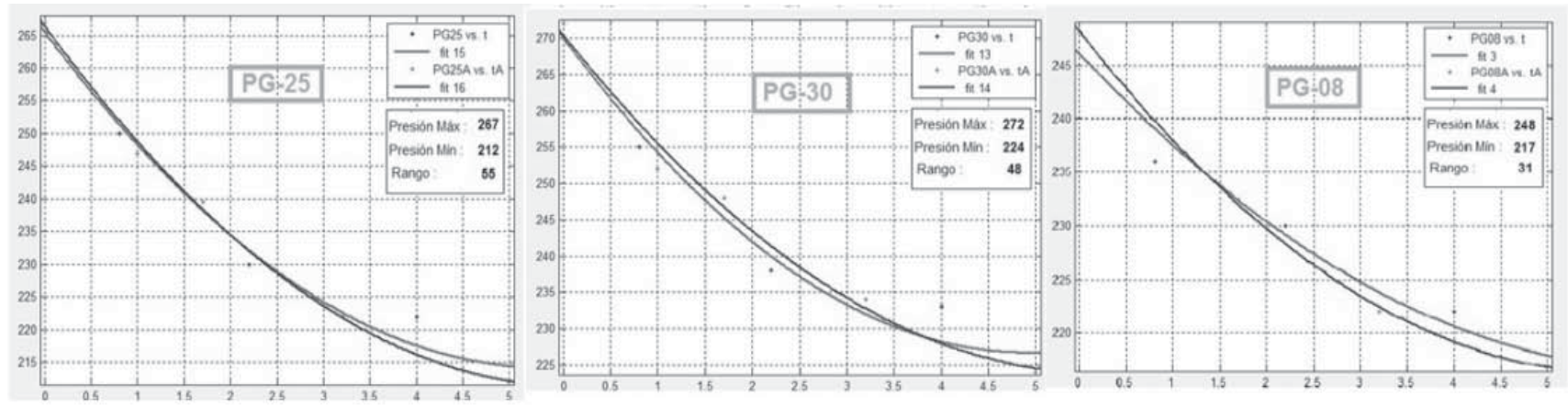

Figura 4. Aproximaciones de las presiones de los cilindros de los pozos PG-25, PG-30 y PG-08.

Con base en los resultados obtenidos y de acuerdo a los valores máximo y mínimo de presión del cilindro, ingresados al programa por el usuario, se obtuvo la gráfica aproximada de presión del cilindro para el pozo DT-38 el día 09 de noviembre de 2009. La gráfica de presión obtenida es la que se observa en la figura 5. 


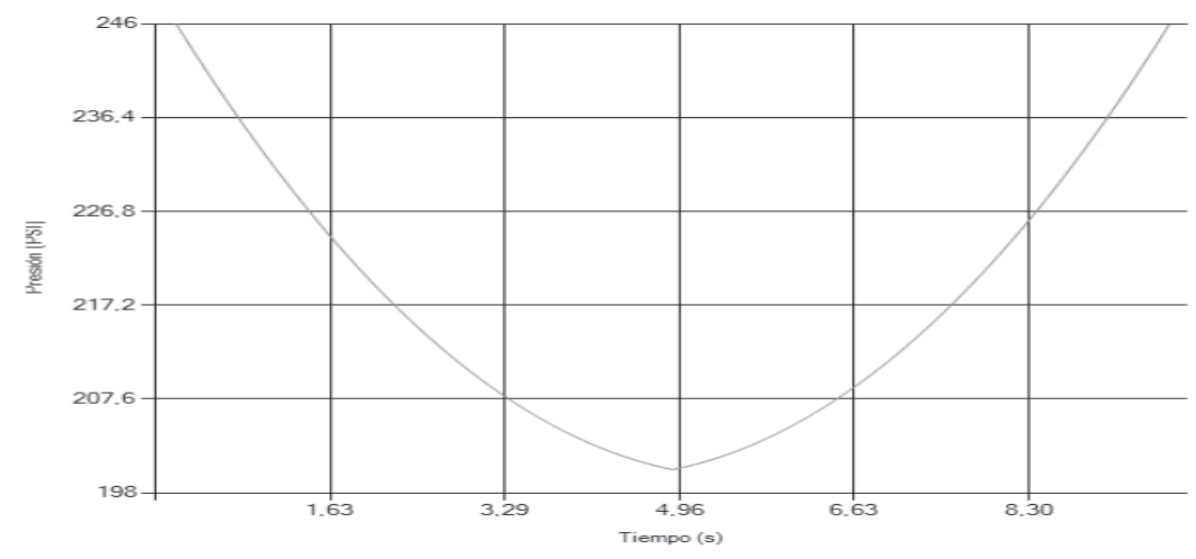

Figura 5. Gráfica de presión del cilindro del pozo DT-38

Con la gráfica de corriente ideal o ajustada y la gráfica de presión del cilindro, el software desarrollado las relaciona mediante el tiempo, el cual es proporcional a la posición de la barra lisa. Luego calcula la presión promedio actual de contrabalanceo y de acuerdo a los picos de corriente actuales y los deseados, se programó de tal forma que el mismo software desarrollado decida qué valor de presión o en que rango debe estar dicha presión para lograr un balanceo muy aproximado de la unidad de bombeo.

Con el fin de validar el software desarrollado y debido a ciertos inconvenientes que se dieron para la realización de la última visita para validar y medir la eficiencia del programa y del método hallado se procedió a hacer la validación de los mismos de una forma alternativa como se describe a continuación.

Primero se procedió a obtener datos de corriente y presión del pozo DT-38 antes y después del balanceo. Esto se obtuvo del software piloto de monitoreo con el que cuenta ECOPETROL actualmente. Se observaron todos los datos de corriente del motor y presión del cilindro, momentos antes en que la unidad se detuvo por desbalanceo e igualmente se capturaron los datos después de balanceada la unidad nuevamente., tal y como se puede observar y analizar en la figura 6. Primero se muestra la gráfica de corriente del motor, la cual indica el comportamiento de la corriente durante todo un día de trabajo y en el cual se detuvo la unidad de bombeo debido al calentamiento del bobinado del motor como consecuencia de una alta corriente que circulaba por el mismo. Igualmente se muestra la gráfica de la presión del cilindro con el fin de analizar su comportamiento en el mismo tiempo de trabajo del motor.

Cabe destacar que la gráfica correspondiente a la corriente cuando la unidad esta desbalanceada, se obtuvo mediante el sensor de potencia del equipo Well Analyzer, la cual se muestra en la parte izquierda de la figura 7. pero por cuestiones de ingreso al pozo no se pudo obtener la gráfica de corriente balanceada con el sensor de potencia del equipo Well Analyzer, ya que la unidad se detuvo y fue balanceada cerca de las 10 p.m., hora en la que solo los recorredores y supervisores tienen permiso para ingresar y manipular el pozo y sus elementos. Razón por la cual se optó por graficar en Excel dicha gráfica balanceada mediante datos arrojados por el sistema de monitoreo de pozos de ECOPETROL, la cual se muestra en la parte derecha de la figura 7.
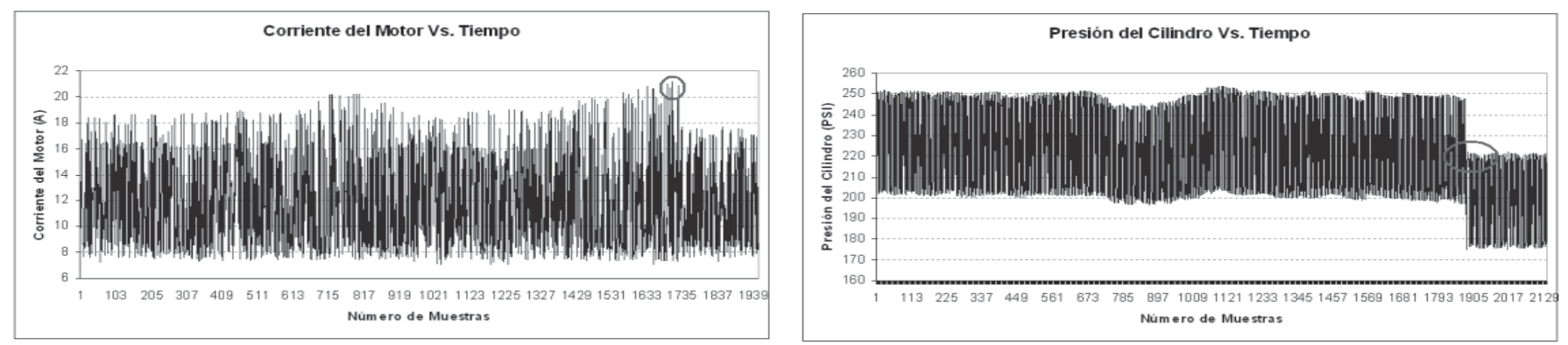

Figura 6. Corriente del motor y presión del cilindro del pozo DT-38 durante un día de trabajo 
Se deduce entonces que la corriente máxima del motor en el momento del desbalanceo es 21 A como se indica en el círculo de la parte izquierda de la figura 6 y la corriente máxima del motor luego de balanceada la unidad es 18 A.

Se analizaron dichos datos para obtener una gráfica aproximada de un ciclo de bombeo momentos después cuando fue nuevamente balanceada la unidad de bombeo, como se ilustra en la parte derecha de la figura 7. Esto con el objetivo de introducir esta nueva gráfica aproximada de corriente al software desarrollado y poder observar el resultado obtenido.

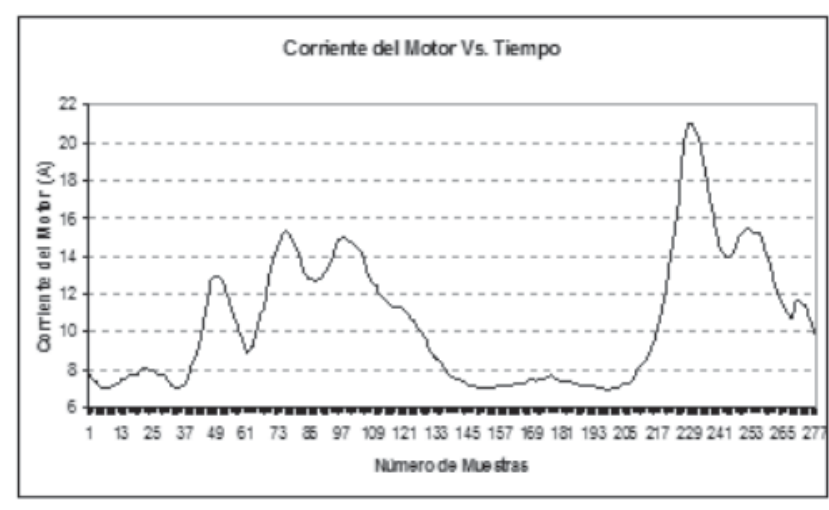

Corriente del motor fuera de balance

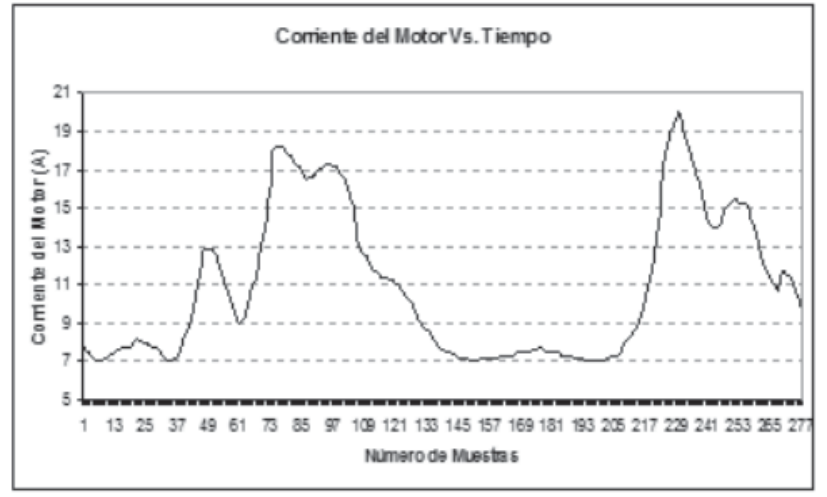

Corriente del motor balanceado

Figura 7. Corriente del motor fuera de balance y corriente del motor balanceado del pozo DT-38

Al igual que con los datos de corriente, se procedió a graficar los datos de presión del cilindro de la unidad de bombeo con el objetivo de analizar su comportamiento en el transcurso del día, como se muestra en la parte derecha de la figura 6. Esta gráfica ilustra el comportamiento de la presión; muestra la unidad balanceada, desbalanceada y nuevamente balanceada.

Se deduce que la presión máxima en el momento del desbalanceo es 250 PSI y la presión mínima en el momento del desbalanceo es 200PSI y que la presión máxima luego del balanceo 220 PSI y la presión mínima luego del balanceo es 170 PSI como se indica en el círculo en la parte derecha de la figura 6.

Una vez analizadas las gráficas anteriores se procedió a construir un archivo.PWR de potencia con el fin de cargar y graficar estos datos en el software desarrollado. Con el objetivo de obtener el rango de presión para el balanceo sugerido por el software desarrollado y compararlo con la presión que le fue suministrada al cilindro en realidad.

Inicialmente se cargó en el programa el archivo.PWR exportado desde el software TWM en el cual inicialmente se adquiere el dato de potencia, cuando la unidad estaba desbalanceada. Dicho resultado es mostrado en la figura 8. 


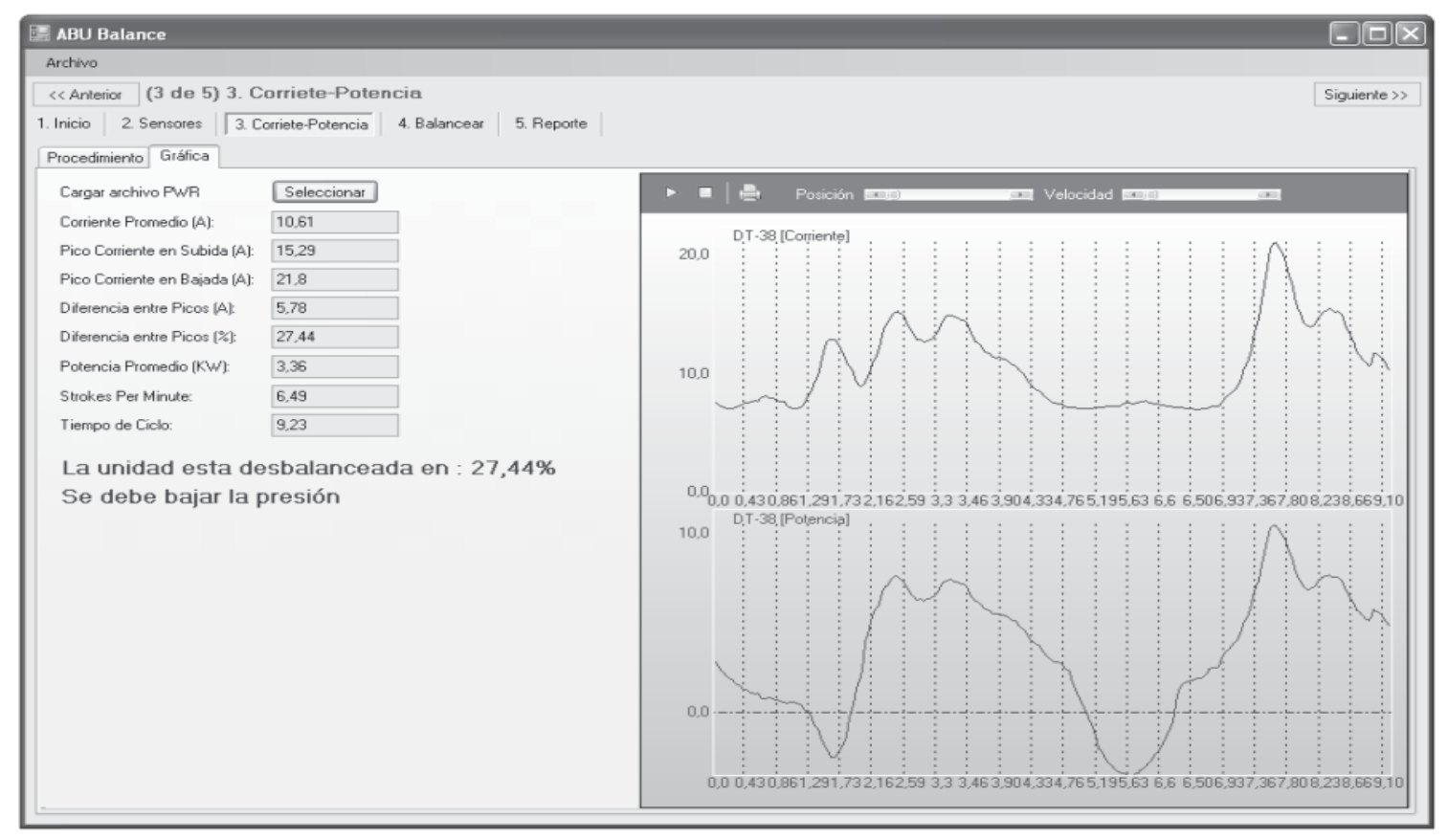

Figura 8. Gráfica de potencia del motor del pozo DT-38 en ABU Balance

Seguidamente se procede al balanceo como tal. El programa sugiere una presión máxima en un rango entre 212 y 222 PSI, Lo cual se acerca al valor de presión máxima que el usuario en el momento le suministró al cilindro que fue de 220 PSI (Figura 9).

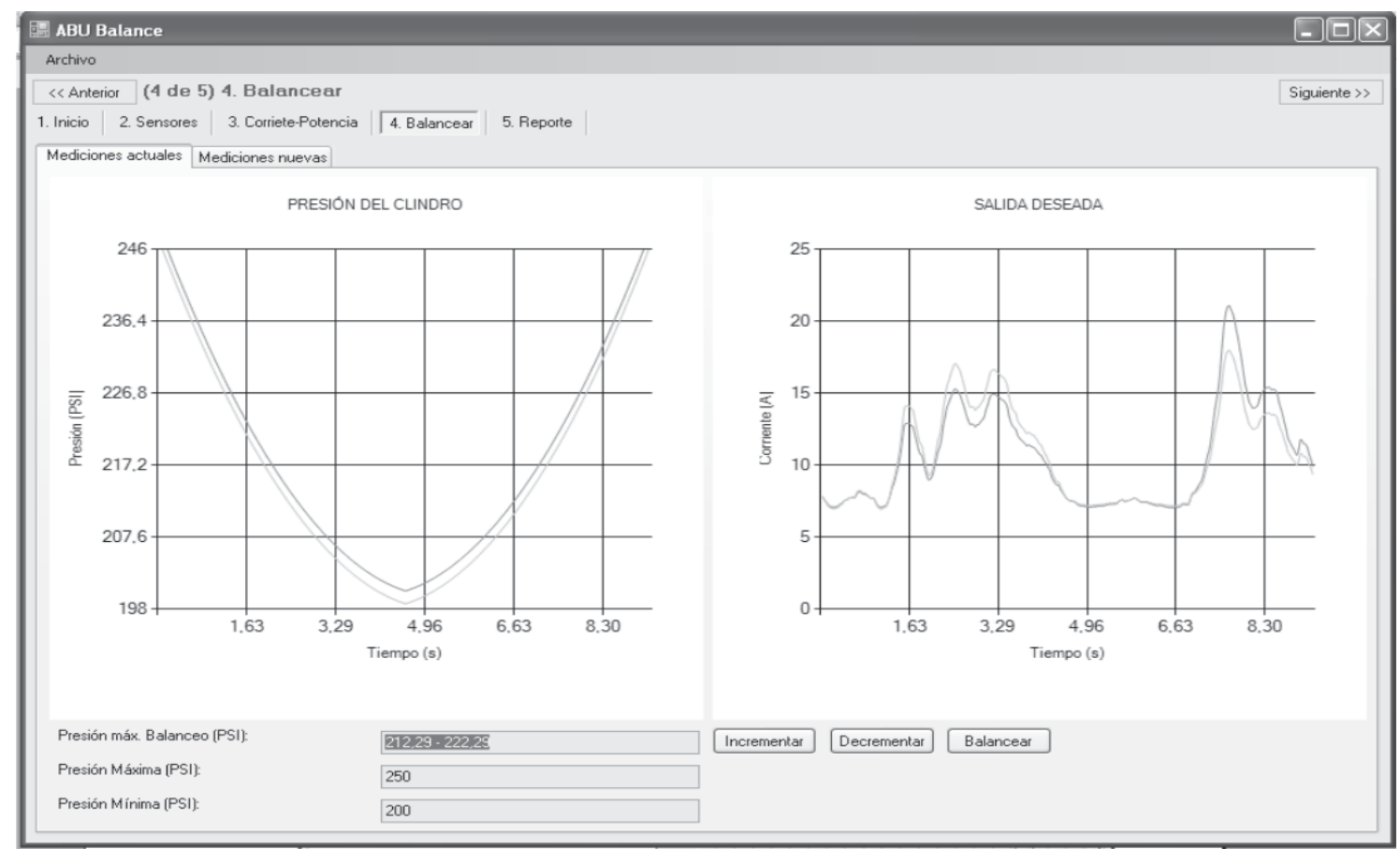

Figura 9. Gráfica de presión del cilindro y corriente del pozo DT-38 en ABU Balance 
Como se observa se obtuvo un rango de presión muy cercano al valor que realmente se le suministró al cilindro para efecto de balancear la unidad y hacer volver a funcionar la unidad de bombeo y no detener la producción por un periodo de tiempo más largo. Aunque el programa no dio un valor de presión exactamente igual al valor real de presión que se le suministró al cilindro, si tuvo una muy buena aproximación.

Finalmente en esta parte de programa una vez la unidad esta balanceada con el nuevo valor de presión sugerido por el ABU Balance, se le pide al usuario volver a tomar la corriente del motor con el sensor de potencia del equipo Well Analyzer y cargar dicha gráfica en esta parte del programa y de esta forma lograr comparar la gráfica de corriente antes y después del balanceo y así determinar la proporción entre la corriente del motor y la presión suministrada al cilindro de la unidad de bombeo y de esta manera verificar un correcto balanceo de la unidad, como se ilustra en la Figura 10.

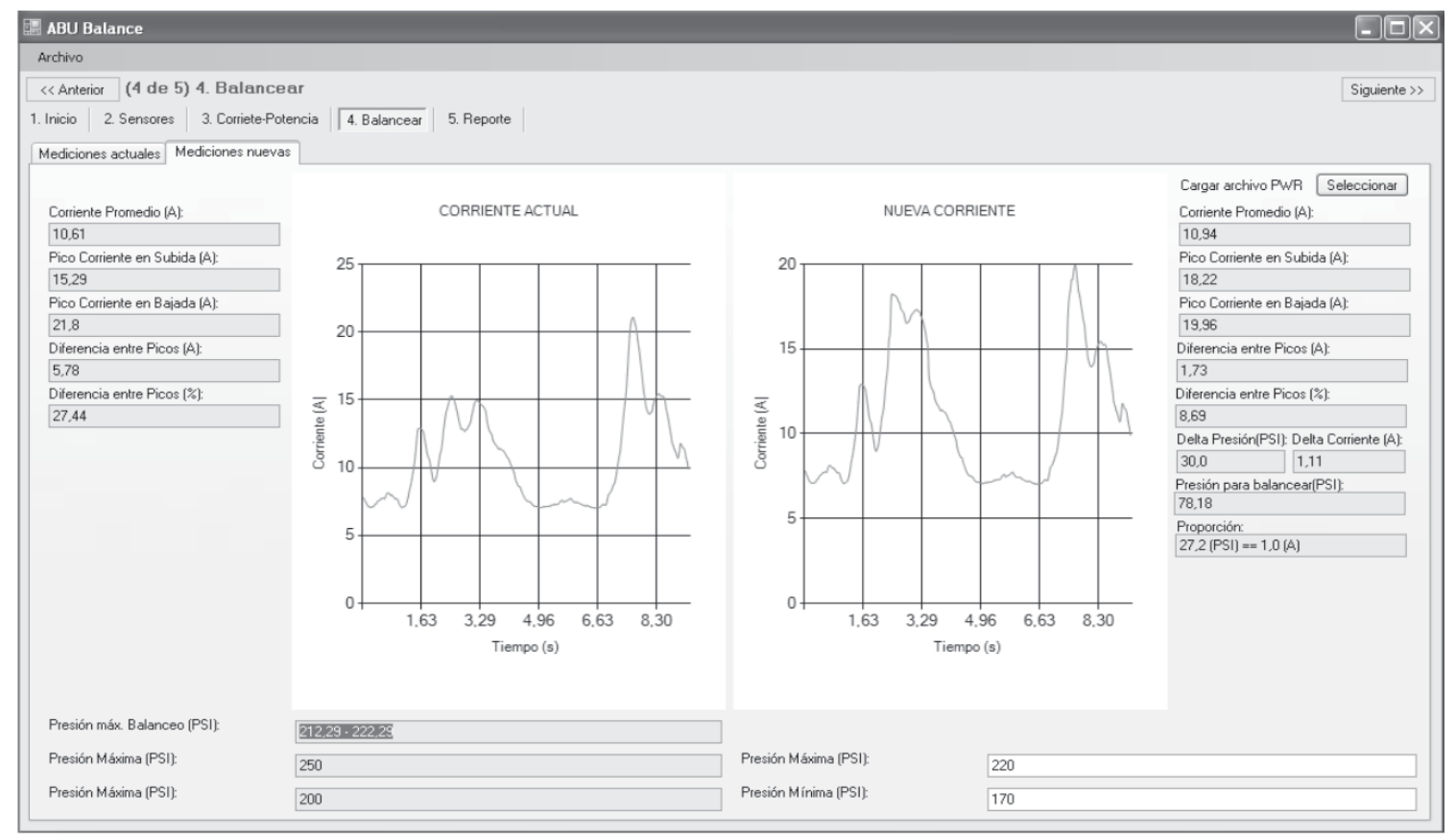

Figura 10. Gráfica antigua y nueva de corriente del motor del pozo DT-38 en ABU Balance

\section{Conclusiones}

Definitivamente el uso del sensor de corriente permitió observar de una manera mucho más fiable, real y exacta los datos de corriente, que son indispensables a la hora de realizar un balanceo amperimétrico, permitiendo realizar mejores análisis para optimizar el proceso de balanceo de unidades del tipo balanceadas por aire en el bombeo mecánico. Adicionalmente permite observar la corriente en todo el tiempo del ciclo de bombeo y de esta manera se puede hacer un mejor análisis y seguimiento de la corriente del motor; cuestión que no era muy optima ya que antes solo se tomaban 4 puntos de corriente en todo el ciclo de bombeo.

Se desarrolló un programa que mediante el seguimientos de cincos pasos, permite balancear de una manera adecuada unidades balanceadas por aire, teniendo en cuenta la parte eléctrica del motor. Dicho programa describe paso a paso la metodología que se debe llevar a cabo para que se pueda llegar a una unidad balanceada y equilibrada permitiendo ahorrar energía, evitando desgastes innecesarios en la unidad y optimizando de cierta manera el proceso de balanceo.

Se logró encontrar una aproximación de la función de presión del cilindro de las unidades de bombeo mecánico balanceadas por aire, aprovechando los datos medidos y utilizando el ajuste de curvas mediante el programa desarrollado, que permitió hacer análisis y ajustes más reales de los que se podrían tener. 
Mediante el programa desarrollado se puede calcular aproximadamente una proporción que existe entre la presión del cilindro que realiza el efecto de contrabalance y la corriente que circula por la unidad motriz de la unidad, y de esta manera claramente determinar un rango de presión que se debe tener en el cilindro para que la unidad trabaje en armonía y se encuentre balanceada.

Es muy importante que estudiantes de Ingeniería Electrónica se introduzcan en diferentes campos tan importantes y productivos como el petrolero, permitiendo que la formación profesional sea mucho más integra y que los estudiantes se puedan mostrar en este medio es un gran reto. Aunque el proceso sea un poco demorado mientras se relaciona y se adapta al medio, es muy gratificante poder contribuir y ayudar a la optimización de procesos que se realizan en una empresa tan importante en nuestra zona y en el país como lo es ECOPETROL.

\section{Referencias Bibliográficas}

1. ECHOMETER COMPANY, 2006. Well Analyzer and TWM Software Operation Manual, REV C. Consultado el 02 de Noviembre de 2010. http://www.echometer.com/support/manuals.

2. LUFKIN INDUSTRIES INC, 2007. Air Balanced Pumping Units Manual. Consultado

el 23 de enero de 2010. http://www.lufkin.com.

3. McCoy, J.N., 1993. Simplified Computer-Aided Analysis of Electrical Current in Motors Used for Beam Pumping Systems. SPE 25447. Presented at the Production Operations Symposium. Oklahoma City, OK.

4. McCoy, J.N., Podio, A.L., Jennings, J., Bill, D., 1992. Motor Power, Current and Torque Analysis to Improve Efficiency of Beam Pumps. Paper presented at the 40th Annual Southwestern Petroleum Short Course.Texas Tech University, Lubbock, TX.

5. Podio, A.L, McCoy, J.N., Collier, F., 1994. Analysis of Beam Pump System Efficiency from Real-Time Measurement of Motor Power. Proceedings of the Third Latin American and Caribbean Petroleum Engineering Conference, Buenos Aires, Argentina.

6. Svinos, J.G., 2005. Optimización de Bombeo Mecánico. Theta Enterprise, Inc. 292 p.

7. Woods, M. D., McCoy J. N,Podio, A.L., 1995. Reducing Electrical Consumption of Beam Pumping Units by Properly Counterbalancing Using PC Software. Paper presented at the 42nd Annual Southwestern Petroleum Short Course. Texas Tech University, Lubbock, TX. 